\title{
60 Years After Castaing's Thesis, What Remains to be Done?
}

\author{
Raynald Gauvin
}

Department of Mining and Materials Engineering, McGill University, 3610 University Street, Montréal, Québec, Canada, H3A 2B2.

2011 is the occasion to celebrate the $60^{\text {th }}$ year of the publication of the famous thesis of Raimond Castaing which constitutes the foundation of quantitative x-ray microanalysis. Despite the impressive achievements of this technique to quantify materials, now at the nanoscale, there are still issues that need to be resolved. In my opinion, the first one is the development of a robust standardless quantitative method to allow performing microanalysis in conventional scanning electron microscope where current measurement and/or stability can be an issue. Also, most of users of scanning electron microscopes are not doing true quantitative x-ray microanalysis because they do not have the time, or the motivation, to insert standards, to acquire spectra and to measure probe current. Even worse, they do not want to learn the basic of x-ray emission. They just want to push the button to get a quick answer. As it is well known pushing the button with the current standardless softwares is the perfect recipes to disaster. The current attitude of the microprobe community in regards to this problem is to say; well standardless does not work, so use standards. We do it, it works so where bordering? Of course, the majority of users are not doing so and by pushing the button, they obtain wrong results that will eventually kill $\mathrm{x}$-ray microanalysis if reliable standardless programs are not developed. The second issue is the development of quantitative methods for non-homogeneous materials, i.e., for materials encountered in real life by real users. It is the opinion of the author that this will happen with the use of correction programs based on Monte Carlo simulations of electron trajectories. Since the computers are getting faster every year, the use of Monte Carlo simulation to perform quantitative $\mathrm{x}$-ray microanalysis has to be taken seriously now.

The problem in the development of standardless quantitative $\mathrm{x}$-ray microanalysis problem is the lack of knowledge with good accuracy of the fundamental parameters of x-ray generation, emission and detection. The most unknown parameter is however the ionization cross-sections $Q_{A}^{E 1}$ of element $\mathrm{A}$ at energy $\mathrm{E}_{1}$. Figure [1] compares experimental values of $\mathrm{Cu} \mathrm{K}$ shell taken from Joy's data base [1] with the recent computations of Bote \& Salvat [2] with that of Casnati [3]. With the scatter of experimental data, it is difficult to tell which model is the most accurate. In his Ph.D. thesis, Gauvin [4] proposed to use the ratio of Cliff-Lorimer $K_{A-B}$ factors measured at 2 different beam energies to determine the $c_{n l}$ parameter of the Bethe equation of ionisation cross-sections. This ratio, $R_{A-B}^{E 1-E 2}$ is equal to:

$$
R_{A-B}^{E 1-E 2}=\frac{K_{A-B}^{E_{2}}}{K_{A-B}^{E_{1}}}=\frac{Q_{B}^{H^{2}} Q_{A}^{L_{1}}}{Q_{B}^{E_{1}^{1}} Q_{A}^{E_{2}^{2}}}
$$

This is exact for the $\mathrm{K}$ lines and for the others lines also above certain energy where the CosterKronig ratio becomes constant. $R_{A-B}^{E 1-E 2}$ can be measured with very good accuracy and measurement of $K_{A-B}$ factors at different beam energy will help to validate good models of ionisation crosssections. Figure [2] shows such a comparison with some of the data of figure [1] and that of C K (not from direct measurement of $K_{A-B}$ factors) with the models of Bote-Salvat [2] and Casnati [3]. 


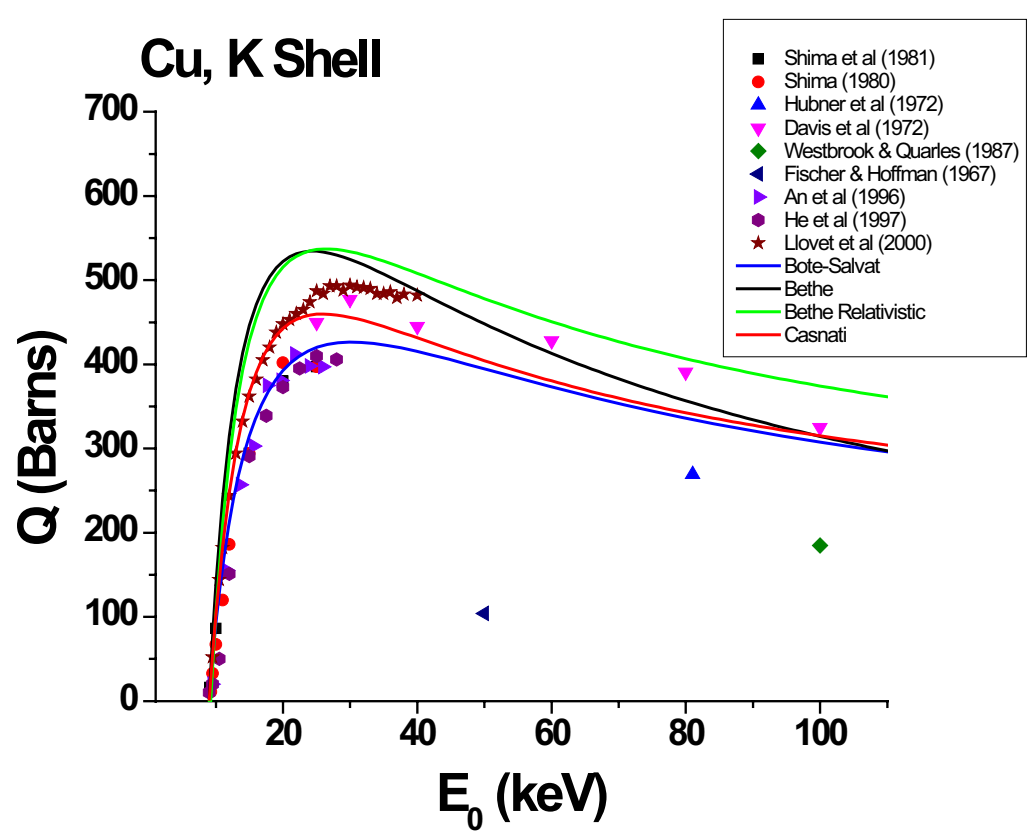

Figure [1] See the text for details.

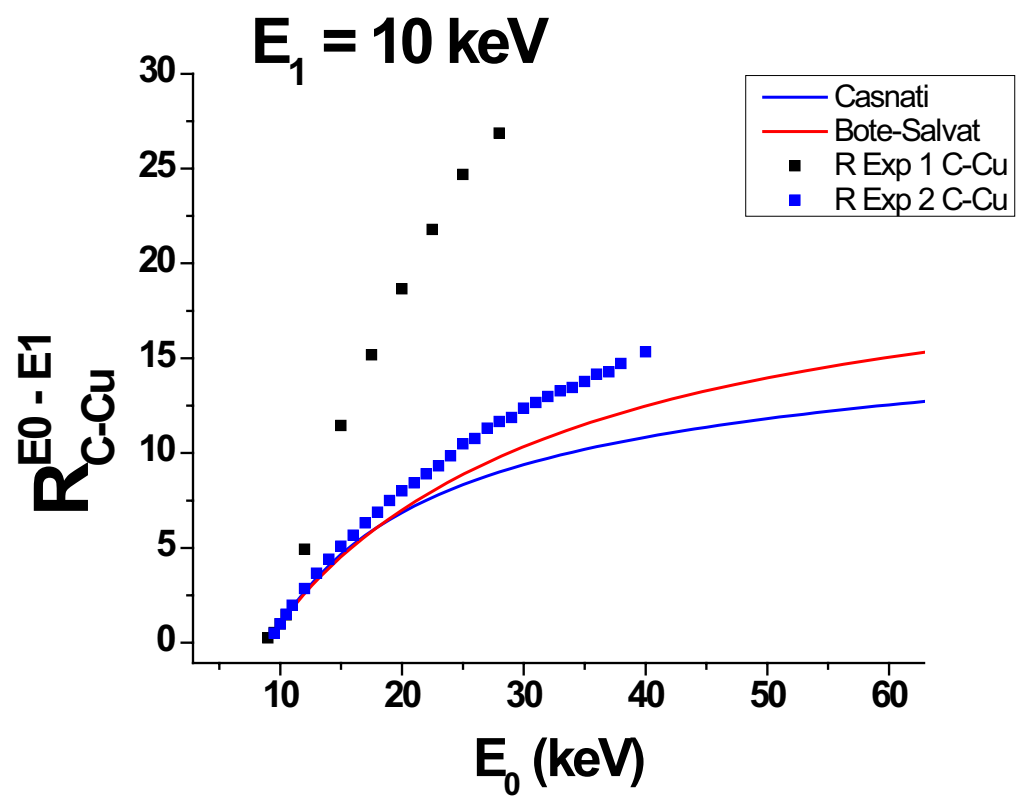

Figure [2] See the text for details.

\section{References}

1. Joy D.C. (2001), Microsc Microanal 7, 159-167.

2. Bote D., Salvat F. (2008), Physical Review, A 77, 042701.

3. Casnati E., Tartari A. and Baraldi C. (1982), J PhysB 15, 155-167.

4. R. Gauvin and G. L'Espérance (1991), Journal of Microscopy 163, pt. 3, 295-306. 